\title{
Who should be "controls" in studies on the neurobiology of psychiatric disorders?
}

\author{
Patricia Boksa, PhD; Ridha Joober, MD, PhD
}

Identifying causal relations is the holy grail of biomedical research. For psychiatric diseases, the quest for "true" causal relations has been very challenging. While causality is a complex philosophical concept, the counterfactual theory of causality (CTC) seems an accepted foundation for causal reasoning in biomedical research. ${ }^{1}$ As an example, under this theory, we assert a causal relation between taking medication $A$ and remission from disease $B$ in a patient $P$ (the factual), if we can convince ourselves that had $\mathrm{P}$ not taken the medication, $\mathrm{P}$ would not have remitted from the disease (the counterfactual). The last part of this statement is said to be "counterfactual," meaning that it is a condition that didn't actually happen. Because it is impossible for patient $\mathrm{P}$ to simultaneously take the medication and not take it, causation remains almost impossible to establish in a specific patient. Causation can only be approached, to variable degrees, using different research designs - the double-blind randomized controlled trial (RCT) being the gold standard. Under an RCT design, the experimental group plays the role of the factual (patients who take the medication), while the control group plays the role of the counterfactual (patients who do not take the medication). Importantly, the random allocation of participants to either group attempts to ensure that the two groups are as identical as possible in all irrelevant variables (e.g., age, sex, education), so the only major difference between the groups is their medication status (see Eichler and colleagues $^{1}$ for an in-depth discussion). Rather than an RCT design, the majority of studies investigating neurobiological correlates of psychiatric disorders use a case-control design, in which it is impossible to randomly allocate participants into the patient or control groups. In the absence of random allocation, it follows that control participants should ideally be identical to patients in all factors, other than the psychiatric disorder under investigation, that might affect the neural outcome being measured. However, an examination of the case-control design in actual practice indicates that we are often quite far from satisfying this criterion.

In this editorial, we examine some of the many factors that are likely to differ between controls and individuals with psychiatric diagnoses and that likely have effects on commonly measured neural outcomes. We conclude by pointing out how the lack of control for many of these confounding factors may influence our understanding of the neurobiology of psychiatric disorders.

\section{Sex, age and medication}

Given substantial evidence in the literature for effects of sex and age on brain structure and function, almost all studies investigating the neurobiology of psychiatric disorders in human populations will control for sex and age by matching or covarying. However, matching for mean age may not be adequate if the range is large. For example, in studies on youth with attention-deficit/hyperactivity disorder (ADHD), it may be problematic to include groups spanning an age range from the prepubertal stage to puberty, a period of life associated with important maturation in different domains, including brain structure and function.

Psychotropic medications have, of course, long been recognized as a potential confound in studies on the neurobiological correlates of psychiatric disorders because these agents are administered with the purpose of changing the course of the disorder and hence its neurobiological underpinnings. To obtain a snapshot of how medication effects are dealt with in studies on neural mechanisms of psychiatric disorders, we examined papers published in JPN in the last two years. Medication was addressed in a wide variety of ways. Some studies controlled for major medication effects in their analyses, while others were less comprehensive, performing separate analyses with a subset of patients taking the same medication or performing exploratory analyses of medication effects. Other studies recruited only patients who were all taking a similar class of medication. Although these approaches may take into account the major medication being used, they do not take into account the common use of polypharmacy or co-medication for adverse effects or for other ancillary problems (e.g., sleep difficulties, anxiety, nonpsychiatric conditions). To avoid the confound of medication

Correspondence to: P. Boksa, Douglas Mental Health University Institute, Dept. of Psychiatry, McGill University, 845 Sherbrooke St W, Montreal, QC H3A 0G4; patricia.boksa@mcgill.ca

DOI: $10.1503 /$ jpn. 180128 
effects, some studies were performed with medication-free patients; however, the "medication-free" period varied from study to study, was often rather short (e.g., 3 months) or was described as medication-free at the time of the study. None of the approaches mentioned above take into account the central nervous sytem (CNS) adaptations likely occurring in response to a changing spectrum of drugs that patients may have been taking over time. Clearly there are no regularly observed conventions on how to address medication effects, and how this is done may be affected by the circumstances of each study. An approach that completely avoids psychotropic medication effects is to investigate medication-naive individuals, but these populations are more difficult to recruit, and investigations are often limited to the very early course of the illness.

Effects of major classes of psychotropic medications (antipsychotics, lithium [Li] and mood stabilizers, antidepressants) on brain structure and function have been investigated to some extent. With regard to structure, in the case of $\mathrm{Li}$ and mood stabilizers, quite consistent evidence has emerged showing that these medications increase grey matter volumes of limbic structures, tending to normalize the opposing effects of bipolar disorder., ${ }^{2,3}$ In contrast, studies to date have not yet shown comparable consistent evidence for dose- and exposure-dependent effects of antipsychotics or antidepressants on brain structure. ${ }^{2,4}$ However, a recent meta-analysis has reported that greater exposure to antipsychotics is associated with decreased parietal lobe and increased basal ganglia volumes, although most of the individual studies did not detect significant associations and were not specifically designed to assess effects of the antipsychotics on brain structure. ${ }^{5}$ This is an issue requiring further methodological refinement. The major classes of psychotropic medications clearly have effects on brain function, as assessed, for example, by functional MRI (fMRI). Consistent research findings are emerging showing effects of antidepressants to reduce limbic activity in response to negative stimuli, effects of mood stabilizers on prefrontal activation and effects of stimulants on attentional circuitry; brain network activation in response to antipsychotic treatment is also a current area of active investigation. ${ }^{6-9}$

\section{Weight, metabolic factors and general health}

Most studies on the neurobiology of psychiatric disorders in clinical populations do not routinely match patients and controls for body weight or body mass index. Yet, heavier weight than that of the general population has been well documented in many populations with psychiatric diagnoses, including schizophrenia (even at very early stages of the disorder), bipolar disorder, major depressive disorder (both increases and decreases) and ADHD. ${ }^{10-14}$ Obesity or overweight have been shown in a number of studies to be associated with reduced volumes of various brain structures and with poorer cognitive outcomes in healthy control individuals, ${ }^{10,15}$ suggesting that weight may be a confounding factor when assessing brain measures in many psychiatric populations. Obesity and even weight gain within the normal range are also associated with increased risk for metabolic abnormalities such as type 2 diabetes, ${ }^{16}$ while type 2 diabetes is itself associated with poorer neuropsychological task performance and functional brain alterations in fMRI studies. ${ }^{17}$ Interestingly, recent studies of adolescents with bipolar disorders or young adults at risk for psychosis have indicated that greater body mass index may have different effects on brain structure in these clinical groups than in controls. ${ }^{11,18}$

That individuals with psychiatric disorders suffer from poorer general health than the rest of the population is supported by several studies demonstrating a 10- to 25-year gap in life expectancy in individuals with psychiatric disorders. ${ }^{19,20}$ These studies indicate that patients die from increased incidences of cardiovascular diseases, cancers (especially lung cancer), chronic obstructive pulmonary disease (COPD) and infections such as influenza and pneumonia. Metabolic syndrome is also much more prevalent in several psychiatric populations, including those with schizophrenia or mood disorders. ${ }^{21,22} \mathrm{~A}$ recent meta-analysis indicates that up to one-third of patients with schizophrenia fulfill criteria for metabolic syndrome, ${ }^{20}$ while metabolic syndrome has been associated with brain structural alterations such as changes in white matter integrity and, more recently, cortical thickness. ${ }^{23,24}$ The presence of a chronic inflammatory state that accompanies metabolic syndrome as well as higher incidence of infection, COPD and poorer dental health among individuals with some psychiatric disorders may provide important confounds in studies on inflammatory or immune mechanisms in the pathophysiology of the psychiatric disorder per se. Yet, while "medical conditions" are sometimes mentioned as an exclusion criterion in studies investigating neural mechanisms of psychiatric conditions, the precise conditions excluded are often quite limited and may not include many conditions (or recent histories of physical illness) that may impact brain function and biochemistry.

\section{Lifestyle factors: sleep; physical activity; smoking; and alcohol, cannabis and other substance use}

Here we discuss a few examples of some common lifestyle habits known to affect neural function and that may differ between psychiatric patients and controls. Sleep disturbances are very highly prevalent among many psychiatric populations. For example, the prevalence of sleep disturbances is estimated to be up to $80 \%$ in individuals with schizophrenia-spectrum disorders, $80 \%$ in those with major depression, $40 \%-90 \%$ during various phases of bipolar disorder, $80 \%$ in those with autism and $50 \%-75 \%$ in those with ADHD. ${ }^{25-28}$ More objective evidence of sleep alterations has been provided by recent meta-analyses of controlled polysomnographic studies documenting alterations in sleep characteristics in schizophrenia; major depression; and anxiety, eating, pervasive developmental, borderline personality and antisocial personality disorders. ${ }^{29,30}$ There is emerging evidence that sleep quality alone has measurable effects on brain structure and function in healthy humans. Poor sleep quality is reported to be associated with cortical atrophy and 
alterations in brain network activity modulating cognitive control and memory in both older and middle-aged adults and with executive dysfunction in adolescents. ${ }^{31-33}$ Sleep has also been investigated as a modulator of CNS function in psychiatric populations. For example, both subjective and objective sleep measures have been reported to modulate dorsal anterior cingulate activity related to emotion regulation in individuals with anxiety and depressive disorders. ${ }^{34}$ For some disorders, such as major depression and ADHD, sleep abnormalities have been hypothesized to be an intrinsic part of the disease pathophysiology; 35,36 however, it is difficult to disentangle this from sleep disturbances due to factors other than the psychiatric disorder per se (e.g., anxiety, medications, substance use, medical conditions, lack of daily occupation, boredom). Nonetheless, sleep quality appears to be an important potential confounding factor in studying CNS alterations responsible for many psychiatric disorders.

Several recent meta-analyses have concluded that people with several major mental illnesses (e.g., major depression, bipolar disorder, schizophrenia and the ultra-high risk state) engage in significantly lower levels of general physical activity than do controls. ${ }^{37-40}$ Effects of physical activity on brain structure in healthy adults have been investigated mainly in older populations, showing that lower levels of physical activity are associated with decreased grey matter volume, especially in the hippocampus. ${ }^{41-43}$ An association between objective physical activity and hippocampal volume has also recently been found in a large population consisting of adults with a greater age range, 40-69 years. ${ }^{42}$ At a functional level, higher fitness or activity levels have also been associated with better cognitive and executive function and activation of brain regions involved in these processes. ${ }^{44}$ Study of the effects of exercise on neurobiological measures in individuals with mental disorders, such as major depression, are only beginning to emerge. ${ }^{45}$ However, physical activity appears to be another lifestyle factor that may influence CNS processes in individuals with psychiatric illness, whether it is considered either a confounding factor or a therapeutic modality.

The ingestion of various substances, such as cigarette smoke, alcohol, cannabis and substances of abuse are another category of lifestyle factors known to affect brain function. A previous editorial in this journal outlined how rates of cigarette smoking are markedly higher (2-5 times) among people with many psychiatric illnesses than the general population and discussed some of the research showing brain changes attributable to smoking. ${ }^{46}$ Effects of regular use of cannabis, alcohol or other substances on human brain structure and function have been quite well described in the literature, ${ }^{47,48}$ while epidemiological studies indicate high rates of substance use among people with psychiatric illness. ${ }^{49,50} \mathrm{Al}-$ though a history of substance use disorder (SUD) is fairly frequently (but not always) an exclusion criterion in studies on the neurobiology of psychiatric disorders, the issue of substance use likely remains a confound of concern. Detecting a history of substance use usually relies on self-report and recall, and may be liable to underreporting. Moreover, while a frank diagnosis of SUD may lead to exclusion, substance use patterns not warranting a diagnosis may have important effects on CNS parameters. For example, neuroimaging studies of binge or heavy episodic drinking in young adults (which may not be categorized as an SUD) have been reported to result in structural alterations in grey and white matter and altered fMRI responses to tasks of executive function. ${ }^{51}$

\section{Psychosocial factors}

Finally, some psychosocial factors may result from having a serious chronic illness and affect indivduals with psychiatric disorders more than controls. Such factors include lack of employment or educational opportunities, lack of a regular routine or meaningful activities, lack of social connection or support and the psychological repercussions resulting from these gaps. To discuss just one example of such psychosocial stressors, there is substantial evidence for an association between loneliness (perceived social isolation) and poorer mental health and for loneliness as a challenge among individuals with many mental disorders. ${ }^{52,53}$ Interestingly, there is emerging research on the neurobiology of loneliness indicating associations between loneliness scores and altered regional grey matter volumes and white matter densities ${ }^{54,55}$ as well as altered functional network activity in brain regions related to social connectedness and vigilance to social threat in lonely individuals. ${ }^{56-58}$ It has been recognized that some individuals will turn to the Internet to alleviate loneliness or boredom, ${ }^{59}$ and there is also significant evidence for effects of excessive Internet use on neurobiological parameters, especially as assessed by brain imaging. ${ }^{60}$ While loneliness might be considered as both a consequence of and a contributor to poorer mental health, it serves as an interesting example of a psychosocial factor whose effects on neurobiology might interact with pathophysiological mechanisms inherent to the disorder.

\section{Conclusion}

When seeking causes for psychiatric disorders, the main object of investigation is the brain, which is both affected by the disorder and is in constant interaction with the environment and with the patients' perceptions of themselves and others. For the majority of psychiatric disorders, the literature provides clear evidence that, had the patients not been afflicted with the disorder under investigation (counterfactual), they would be less likely to be taking medications, to smoke, to be overweight and to consume drugs of abuse. They would also be more likely to be physically active, to be in good physical health, to have good sleep quality and to have regular occupations and supportive social circles. There is also strong evidence that these characteristics produce measurable brain effects on their own, regardless of the disorder under investigation. It follows, then, that these characteristics, and possibly many others, should be matched between patients and controls if we want to assert causal relations between diseases and specific neural outcome measures under investigation. Unfortunately, this is very hard to achieve. The capacity of big data to incorporate a large number of variables into analyses may make statistical control of the main confounders more 
feasible with time. However, this will still require careful and comprehensive experimental design and comes with a price (i.e., the need for much larger sample sizes and consequent difficulty in recruiting patients and controls). Another approach sometimes used in an attempt to match for lifestyle factors is the use of patient controls. However, this approach carries its own complexities (e.g., the choice of which patients to select as controls and which characteristics to match for).

With the advent of highly sensitive tools and techniques, we are now able to detect alterations in human brain structure and function in response to quite slight variations in internal and external cues and conditions. Given how numerous these conditions are, it raises the question of how large the signal-to-noise ratio might be for us to detect brain changes that are actually integral to the pathophysiology of a psychiatric disorder in question. At the very least, it might be useful to keep in mind that often our experiments with humans in all their complexity fall short of investigating the inherent neurobiology of a particular psychiatric disorder, but may provide a window on a combination of the neurobiology of a disorder plus its physical, psychological and social consequences.

Affiliations: From the Douglas Mental Health University Institute, Dept. of Psychiatry, McGill University, Montreal, Que., Canada.

Competing interests: See jpn.ca/about/ for editorial board member competing interests.

\section{References}

1. Eichler HG, Bloechl-Daum B, Bauer P, et al. "Threshold-crossing": A useful way to establish the counterfactual in clinical trials? Clin Pharmacol Ther 2016;100:699-712.

2. McDonald C. Brain structural effects of psychopharmacological treatment in bipolar disorder. Curr Neuropharmacol 2015;13:445-57.

3. Hajek T, Kopecek M, Höschl C, et al. Smaller hippocampal volumes in patients with bipolar disorder are masked by exposure to lithium: a meta-analysis. J Psychiatry Neurosci 2012;37:333-43.

4. Roiz-Santiañez R, Suarez-Pinilla P, Crespo-Facorro B. Brain structural effects of antipsychotic treatment in schizophrenia: a systematic review. Curr Neuropharmacol 2015;13:422-34.

5. Huhtaniska S, Jääskeläinen E, Hirvonen N, et al. Long-term antipsychotic use and brain changes in schizophrenia - a systematic review and meta-analysis. Hum Psychopharmacol 2017;32:e2574.

6. Wessa M, Lois G. Brain functional effects of psychopharmacological treatment in major depression: a focus on neural circuitry of affective processing. Curr Neuropharmacol 2015;13:466-79.

7. Wandschneider B, Koepp MJ. Pharmaco fMRI: determining the functional anatomy of the effects of medication. Neuroimage Clin 2016;12:691-7.

8. Laidi C, Houenou J. Brain functional effects of psychopharmacological treatments in bipolar disorder. Eur Neuropsychopharmacol 2016;26:1695-740.

9. De Rossi P, Chiapponi C, Spalletta G. Brain functional effects of psychopharmacological treatments in schizophrenia: a networkbased functional perspective beyond neurotransmitter systems. Curr Neuropharmacol 2015;13:435-44.

10. Minichino A, Ando A, Francesconi M, et al. Investigating the link between drug-naive first episode psychoses (FEPs), weight gain abnormalities and brain structural damages: Relevance and implications for therapy. Prog Neuropsychopharmacol Biol Psychiatry 2017; 77:9-22.

11. Islam AH, Metcalfe AWS, MacIntosh BJ, et al. Greater body mass index is associated with reduced frontal cortical volumes among adolescents with bipolar disorder. J Psychiatry Neurosci 2018; 43:120-30.
12. Paans NPG, Bot M, Gibson-Smith D, et al. Which biopsychosocial variables contribute to more weight gain in depressed persons? Psychiatry Res 2017;254:96-103.

13. Gibson-Smith D, Bot M, Milaneschi Y, et al. Major depressive disorder, antidepressant use, and subsequent 2-year weight change patterns in the Netherlands Study of Depression and Anxiety. J Clin Psychiatry 2016;77:e144-51.

14. Cortese S, Tessari L. Attention-deficit/hyperactivity disorder (ADHD) and obesity: update 2016. Curr Psychiatry Rep 2017;19:4.

15. Willette AA, Kapogiannis D. Does the brain shrink as the waist expands? Ageing Res Rev 2015;20:86-97.

16. Feldman AL, Griffin SJ, Ahern AL, et al. Impact of weight maintenance and loss on diabetes risk and burden: a population-based study in 33,184 participants. BMC Public Health 2017;17:170.

17. Macpherson H, Formica M, Harris E, et al. Brain functional alterations in type 2 diabetes - a systematic review of fMRI studies. Front Neuroendocrinol 2017;47:34-46.

18. Koivukangas J, Björnholm L, Tervonen O, et al. Body mass index and brain white matter structure in young adults at risk for psychosis - The Oulu Brain and Mind Study. Psychiatry Res Neuroimaging 2016;254:169-76.

19. Lawrence D, Hancock KJ, Kisely S. The gap in life expectancy from preventable physical illness in psychiatric patients in Western Australia: retrospective analysis of population based registers. BMJ 2013;346:f2539.

20. Olfson M, Gerhard T, Huang C, et al. Premature mortality among adults with schizophrenia in the United States. JAMA Psychiatry 2015;72:1172-81.

21. Mitchell AJ, Vancampfort D, Sweers K, et al. Prevalence of metabolic syndrome and metabolic abnormalities in schizophrenia and related disorders - a systematic review and meta-analysis. Schizophr Bull 2013;39:306-18.

22. Yamagata AS, Mansur RB, Rizzo LB, et al. Selfish brain and selfish immune system interplay: a theoretical framework for metabolic comorbidities of mood disorders. Neurosci Biobehav Rev 2017;72:43-9.

23. Alfaro FJ, Gavrieli A, Saade-Lemus P, et al. White matter microstructure and cognitive decline in metabolic syndrome: a review of diffusion tensor imaging. Metabolism 2018;78:52-68.

24. Schwarz NF, Nordstrom LK, Pagen LHG, et al. Differential associations of metabolic risk factors on cortical thickness in metabolic syndrome. Neuroimage Clin 2017;17:98-108.

25. Klingaman EA, Palmer-Bacon J, Bennett ME, et al. Sleep disorders among people with schizophrenia: emerging research. Curr Psychiatry Rep 2015;17:79.

26. Murphy MJ, Peterson MJ. Sleep disturbances in depression. Sleep Med Clin 2015;10:17-23.

27. Gold AK, Sylvia LG. The role of sleep in bipolar disorder. Nat Sci Sleep 2016;8:207-14.

28. Ramtekkar UP. DSM-5 changes in attention deficit hyperactivity disorder and autism spectrum disorder: implications for comorbid sleep issues. Children (Basel) 2017;4.pii:E62.

29. Baglioni C, Nanovska S, Regen W, et al. Sleep and mental disorders: a meta-analysis of polysomnographic research. Psychol Bull 2016;142:969-90.

30. Winsper C, Tang NK, Marwaha S, et al. The sleep phenotype of borderline personality disorder: a systematic review and metaanalysis. Neurosci Biobehav Rev 2017;73:48-67.

31. Scullin MK. Do older adults need sleep? A review of neuroimaging, sleep, and aging studies. Curr Sleep Med Rep 2017;3:204-14.

32. Del Brutto OH, Mera RM, Zambrano M, et al. The association between poor sleep quality and global cortical atrophy is related to age. Results from the Atahualpa Project. Sleep Sci 2016;9:147-50.

33. Clark DB, Chung T, Martin CS, et al. Adolescent executive dysfunction in daily life: relationships to risks, brain structure and substance use. Front Behav Neurosci 2017;11:223.

34. Klumpp H, Roberts J, Kapella MC, et al. Subjective and objective sleep quality modulate emotion regulatory brain function in anxiety and depression. Depress Anxiety 2017;34:651-60.

35. Zaki NFW, Spence DW, BaHammam AS, at al. Chronobiological theories of mood disorder. [Epub ahead of print]. Eur Arch Psychiatry Clin Neurosci 2017;10.1007/s00406-017-0835-5.

36. Dueck A, Berger C, Wunsch K, et al. The role of sleep problems and circadian clock genes in attention-deficit hyperactivity disorder and mood disorders during childhood and adolescence: an update. J Neural Transm (Vienna) 2017;124(Suppl 1):127-38. 
37. Schuch F, Vancampfort D, Firth J, et al. Physical activity and sedentary behavior in people with major depressive disorder: a systematic review and meta-analysis. J Affect Disord 2017;210:139-50.

38. Vancampfort D, Firth J, Schuch F, et al. Physical activity and sedentary behavior in people with bipolar disorder: a systematic review and meta-analysis. J Affect Disord 2016;201:145-52.

39. Stubbs B, Firth J, Berry A, et al. How much physical activity do people with schizophrenia engage in? A systematic review, comparative meta-analysis and meta-regression. Schizophr Res 2016; 176:431-40.

40. Carney R, Cotter J, Bradshaw T, et al. Cardiometabolic risk factors in young people at ultra-high risk for psychosis: a systematic review and meta-analysis. Schizophr Res 2016;170:290-300.

41. Erickson KI, Leckie RL, Weinstein AM. Physical activity, fitness, and gray matter volume. Neurobiol Aging 2014;35(Suppl 2):S20-8.

42. Hamer M, Sharma N, Batty GD. Association of objectively measured physical activity with brain structure: UK Biobank study. [Epub ahead of print]. J Intern Med 2018;10.1111/joim.12772.

43. Papenberg G, Ferencz B, Mangialasche F, et al. Physical activity and inflammation: effects on gray-matter volume and cognitive decline in aging. Hum Brain Mapp 2016;37:3462-73.

44. Stillman CM, Erickson KI. Physical activity as a model for health neuroscience. Ann N Y Acad Sci 2018 May 6. doi: 10.1111/nyas.13669.

45. Heinzel S, Rapp MA, Fydrich T, et al. Neurobiological mechanisms of exercise and psychotherapy in depression: the SPeED study-rationale, design, and methodological issues. Clin Trials 2018;15:53-64.

46. Boksa P. Smoking, psychiatric illness and the brain. J Psychiatry Neurosci 2017:42:147-9.

47. Zahr NM, Pfefferbaum A. Alcohol's effects on the brain: neuroimaging results in humans and animal models. Alcohol Res 2017;38:183-206.

48. Weinstein A, Livny A, Weizman A. Brain imaging studies on the cognitive, pharmacological and neurobiological effects of cannabis in humans: evidence from studies of adult users. Curr Pharm Des 2016;22:6366-79.
49. Moore E, Mancuso SG, Slade T, et al. The impact of alcohol and illicit drugs on people with psychosis: the second Australian National Survey of Psychosis. Aust N Z J Psychiatry 2012;46:864-78.

50. Toftdahl NG, Nordentoft M, Hjorthøj C. Prevalence of substance use disorders in psychiatric patients: a nationwide Danish population-based study. Soc Psychiatry Psychiatr Epidemiol 2016;51:129-40.

51. Cservenka A, Brumback T. The burden of binge and heavy drinking on the brain: effects on adolescent and young adult neural structure and function. Front Psychol 2017;8:1111.

52. Leigh-Hunt N, Bagguley D, Bash K. at al. An overview of systematic reviews on the public health consequences of social isolation and loneliness. Public Health 2017;152:157-71.

53. Mann F, Bone JK, Lloyd-Evans B, et al. A life less lonely: the state of the art in interventions to reduce loneliness in people with mental health problems. Soc Psychiatry Psychiatr Epidemiol 2017;52:627-38.

54. Kong X, Wei D, Li W, et al. Neuroticism and extraversion mediate the association between loneliness and the dorsolateral prefrontal cortex. Exp Brain Res 2015;233:157-64.

55. Nakagawa S, Takeuchi H, Taki Y, et al. White matter structures associated with loneliness in young adults. Sci Rep 2015;5:17001.

56. Cacioppo S, Capitanio JP, Cacioppo JT. Toward a neurology of loneliness. Psychol Bull 2014;140:1464-504

57. Layden EA, Cacioppo JT, Cacioppo S, et al. Perceived social isolation is associated with altered functional connectivity in neural networks associated with tonic alertness and executive control. Neuroimage 2017;145(Pt A):58-73.

58. Inagaki TK, Muscatell KA, Moieni M, et al. Yearning for connection? Loneliness is associated with increased ventral striatum activity to close others. Soc Cogn Affect Neurosci 2016;11:1096-101

59. Nowland R, Necka EA, Cacioppo JT. Loneliness and social internet use: pathways to reconnection in a digital world? Perspect Psychol Sci 2018;13:70-87.

60. Yao YW, Liu L, Ma SS, et al. Functional and structural neural alterations in Internet gaming disorder: A systematic review and metaanalysis. Neurosci Biobehav Rev 2017;83:313-24. 